\title{
CONCEPTO DE CLASE DE EDUCACIÓN FÍSICA EN LOS ESTUDIANTES DEL PROGRAMA DE LICENCIATURA EN EDUCACIÓN FÍSICA DE LA UNIVERSIDAD DEL QUINDÍO
}

\section{CLASS CONCEPT OF PHYSICAL EDUCATION STUDENTS IN DEGREE PROGRAM IN PHYSICAL EDUCATION AT THE UNIVERSITY OF QUINDIO.}

\author{
Jorge Alberto López García ${ }^{1}$,Johanna Andrea Murillo², Jairo Antonio Sánchez Cadavid, \\ Mauricio Posada Molina', Jhon Hermógenes Galíndez'. \\ ${ }^{1}$ Programa de Lic. Educación Física y Deportes. Universidad del Quindío. Grupo de Investigación Tejiendo Redes. Universidad del Quindío, \\ ${ }^{2}$ Estudiantes Colaboradores
}

Fecha de recibido: Febrero 3 de 2010

Fecha de aceptado: Junio 9 de 2010

Correspondencia: Programa Licenciatura en Educación Física. Universidad del Quindío. Av. Bolivar calle 12 norte Armenia Quindío. Correo electrónico: jorgea319@hotmail.com

\section{RESUMEN}

El objetivo de este estudio fue el de indagar acerca del concepto de "Clase de educación física" que tienen los estudiantes del programa de Licenciatura en Educación Física y Deportes de la Universidad del Quindío el cual, partiendo de la comprensión de ese concepto de clase en los estudiantes que cursan los semestres del I al III de nuestro programa, servirá de referente teórico para apalancar los procesos académicos de nuestro programa o plan curricular, en especial en la búsqueda de ese estado del arte que siempre nos tiene en deuda y sobre todo acerca de la concepción que presenta para nuestros estudiantes la clase de educación física. Este proyecto se enmarca en el terreno de los jóvenes universitarios, ya que la población que hace parte de este estudio está en edades entre los 15 a los 19 años de edad, por lo tanto las nociones o interpretaciones respecto al concepto que tienen los estudiantes frente a la clase de Educación Física, ayuda al rompimiento de concepciones tradicionales de la clase y permite la puesta en discusión de otras miradas que permita pensarse la clase de otra manera. En un contexto como lo es la Universidad y en las edades en que se desarrolló el estudio, se hace de vital importancia y actual, permitir el repensar las practicas y reconocer los imaginarios de los jóvenes de nuestra región en campo de la educación física y los deportes que toman la decisión de participar de nuestro proyecto de vida académico.

Palabras claves: Metodología, Innovación, La clase, Actitud, El ambiente

\begin{abstract}
The aim of this study was to inquire about the concept of "physical education class" students have undergraduate program in physical education and sport at the University of Quindio, which stems from the understanding of the concept of classes in the Students enrolled in semester 1 to 3 of our program, will serve as theoretical reference to leverage the academic processes of our program or plan curriculum, especially the search for the state of the art that always keeps us in debt and especially about of conception that they pose to our students of physical education class. This project is part of the field of university students as the population that is part of this study was aged between 15 and 19 years of age, so the notions or interpretations about the concept that students have in front a physical education class, help the breakdown of traditional conceptions of class and allowed the questioning of other sights to allow the class thought otherwise. In a context as is the University and in the ages at which developed the study, it is vitally important and current practices allow and recognize rethink concepts of those young people of our region in the field of physical education and sports taking the decision to participate in our academic life project.
\end{abstract}

Keywords: Methodology, Innovation, Class, Attitude, The environment 


\section{INTRODUCCIÓN}

En la actualidad existe un interés creciente por preguntarnos acerca de la Educación física y su tradición en nuestros currículos, desde el punto de vista de su concepción y práctica.

La educación física como área del conocimiento ${ }^{3}$, hoy en día mantiene su legitimidad institucional como un campo de operaciones, principalmente en los deportes y diversificaciones, como escape de la alta tensión que se genera al interior de los planteles educativos, desde el hacer, el pensar, el sentir y el vivir la vida extra e intra escolar, de modos diferentes; como una educación animada a la transformación de los sujetos.

Estas transformaciones podrían estar enmarcadas desde varias ópticas: hacia el cuerpo, muestran su fundamentación en los valores sociales; para unos estarán a la orden de lo estético, de la salud, del desarrollo de capacidades físicas especiales, o lo más importante mejorar su calidad de vida y de otro lado, la educación física en ámbito escolar es vista o se convierte en un aparato disciplinatorio, donde el docente encuentra en ella un medio para que el estudiante responda a las demandas que se exigen en las áreas que para ellos son más importantes.

Dada su importancia en la sociedad y particularmente en nuestra cultura educativa, la educación física ha sido objeto de estudio de distintas disciplinas como: La Biología, la Kinesiología, la Fisiología, la antropología, la Historia, la Etnología, la Sociología, la Psicología y las ciencias de la educación. Estudios que han servido para verla como un fenómeno de masas, catalizador de impulsos individuales y grupales o formador de la persona por sus innumerables cualidades, reflejo de los valores de una determinada sociedad y mecanismos de enculturación entre otros.

De manera pues que el área de la educación física se reconoce como una práctica social y de desarrollo humano en un proyecto educativo que privilegia el cultivo de la persona como un ser total en todas sus dimensiones.

\section{PREGUNTA ORIENTADORA}

¿Cuál es el concepto de clase de educación física que tienen los estudiantes del programa de licenciatura en educación física de la universidad del Quindío?. Objetivo del estudio
Indagar acerca del concepto de clase de Educación Física que tienen los estudiantes de I al III semestre del programa de Licenciatura en Educación Física y Deportes de la Universidad del Quindío.

\section{ANÁLISIS CUALITATIVO}

\section{El concepto de clase de Educación física}

"Mi concepto era correr, jugar, estirar; pero ahora he aprendido muchas cosas que hace que la educación física que yo aprendí cambie totalmente". (Estudiante III semestre)

La Educación Física se podría definir como una disciplina pedagógica que potencia la educación del movimiento y de las estructuras motrices que lo hacen posible, utilizando para ello el propio movimiento.

Desde esta perspectiva, la Educación Física por tener el carácter de intervención formativa, en especial sobre la motricidad y el movimiento humano, no puede concebirse como conocimiento puro, sino como un complejo entramado de conocimientos aplicables a la Educación y a la formación del hombre.

Es preciso destacar que para este estudio podemos utilizar indistintamente los conceptos de motricidad, movimiento y actividad física, por entender que son maneras de interpretar distintos niveles organizativos del mismo fenómeno.

El desarrollo de este semillero de investigación al preguntarse sobre "el concepto de clase de educación física en los estudiantes del programa de licenciatura en educación física y deporte de la Universidad del Quindío", encontramos que se organizó y desarrolló en tres momentos: el primero, denominado exploratorio, el segundo configuración y el tercero de reconfiguración.

\section{ELPROCEDIMIENTO}

El estudio se ubica dentro de los paradigmas y métodos cualitativos, los cuales se basan en la comprensión de un fenómeno en particular, más que en la explicación que se le pueda dar a este y desde la perspectiva, en lo social lo enmarcamos en la cuasietnografía ya que el despliegue se hace desde el paradigma interpretativo el cuál se preocupa por la comprensión e interpretación de los fenómenos de una realidad en un tiempo y contexto determinado.

\footnotetext{
${ }^{3}$ La Ley 115 o ley General de Educación de 1994, en su artículo 23, establece cuales son las áreas fundamentales y obligatorias del conocimiento que se deben ofrecer de acuerdo al currículo institucional, encontrando que la educación física es una de ellas y debe formar parte de todos los currículos institucionales en nuestro país.
} 
El estudio acoge el diseño de "Complementariedad etnográfica" propuesto por Murcia y Jaramillo (2000). Un estudio con jóvenes es incomprensible si no se analiza desde el contexto global del grupo que lo ejecuta, desde la estructura manifestada y desde una relación comunicativa que posibilite entender los actos performativos que subyacen en las expresiones tanto fonéticas como corporales, pues es la mirada de la estructura desde el contrato comunicativo, la que lleva a comprender el sentido de esa estructura. Por ello, estos elementos de búsqueda articulada de la realidad se condensarán en tres momentos:

El momento de pre-configuración de la realidad, que muestra una apariencia de la estructura de dicha realidad y está referido al momento donde se adquiere una primera aproximación a la posible estructura sociocultural (pre - estructura). Denominado también como momento exploratorio.

El momento de configuración de la realidad, que inicia con la puesta en escena de una guía flexible que permite orientar la búsqueda de esa estructura. El trabajo de campo al desarrollar este momento, debe realizarse a profundidad (intensivo y extensivo), para obtener una estructura más plausible.

El momento de re - configuración de la realidad encontrada, donde se realiza un análisis de los hallazgos socioculturales desde una triple perspectiva: la perspectiva del investigador, la perspectiva de la teoría formal y la perspectiva de la teoría sustantiva.

\section{TÉCNICAS E INSTRUMENTOS}

Se utilizarán básicamente la observación participante, la entrevista en profundidad y la auto-biografía, sin descuidar otras posibles técnicas como grupos focales de discusión, y posibles eventos que sirvan de ayuda para ubicar los posibles informantes claves.

\section{EL DESARROLLO}

En el primer momento, los estudiantes emplearon la técnica de observación directa sobre el desempeño de los profesores y estudiantes de las diferentes actividades académicas propuestas por el programa. La clase de educación física, gira alrededor de actividades teóricas, teórico prácticas y prácticas, lo que permite enriquecer aún más el proceso de enseñanza aprendizaje de los estudiantes.

Los estudiantes investigadores de este semillero y el tutor, luego de la puesta en escena como observadores, empezamos la tarea de consolidar un diario de campo que recogiera las primeras premisas de la posible pre-estructura y categorías propia de este tipo de investigación.

De este modo, las primeras observaciones realizadas fueron muy generales, desde una óptica externa, pero a medida que se fue asumiendo el rol de investigador, se fue logrando una mejor interacción con la población objeto de este estudio y como resultado de ello, los hallazgos en el diario de campo propuesto giró entorno a:

Pre-estructura:

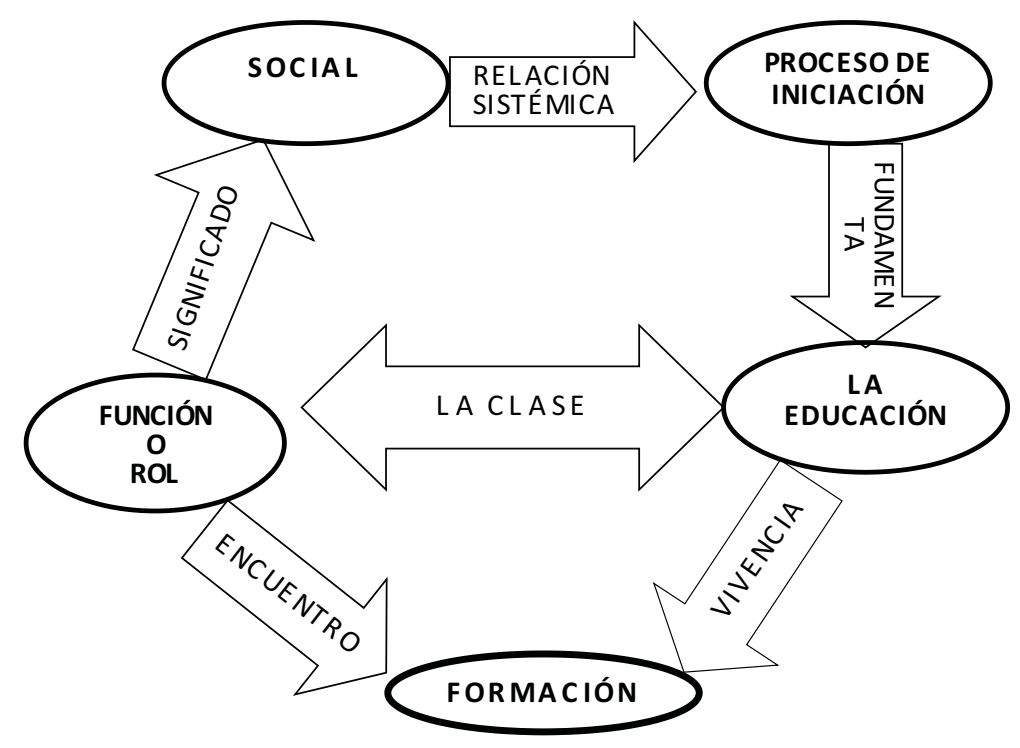

Rev. Invest. Univ. Quindío (21): 87- 94. Armenia - Colombia 
La vivencia: aquí se hizo referencia a la descripción sobre lo que se hace en la clase de educación física, como se organizan los grupos, como es el desempeño de los estudiantes y del profesor hasta como se saludan ellos entre sí y con los demás, así mismo, que hacen mientras están dentro del aula y como se desempeñan fuera de ella.

"Se llega al coliseo, se saludan de mano y con respeto, hola, de beso con las niñas, parece que se comentan los compromisos que tuvieron, los protocolos son algunos alegres y dinámicos... se hace una serie de ejercicios en los que todos participan de forma dinámica, las niñas son calladas, se arreglan para la clase, se comento sobre los próximos temas y algunos hablan mucho "(diario $N^{\circ} 1$, Jhon Galíndez)

Se deja entrever que la clase presenta un desarrollo normal por parte del docente y de los estudiantes; hay buena empatía entre el grupo, claro que ello varía de acuerdo con las vivencias lúdicas y pedagógicas compartidas dentro del ámbito educativo.

Del anterior relato, podemos interpretar que la educación física es un espacio para la formación en valores y como medio de desarrollo integral, canalizador de energías, medio de compensación emocional y de integración social ${ }^{4}$.

El encuentro: el compartir con las personas objeto del trabajo, permite la oportunidad de compartir el escenario propuesto dando una mirada diferente de la premisa de "clase" como lo manifiesta Jaime Andrés Ocampo, un estudiante del programa, al decir:

"En este grupo se ve la variedad de estudiantes, los sindicalistas, los recién salidos de colegio que creen que todavía están hay, los adultos que tiene como mas responsabilidad, los recocheros que quieren coger todo como broma, los que por lo visto no se han dado cuenta de que la carrera no es lo que ellos quieren, ya que se alejan y se esconden detrás de los que participan"

La diversidad de las personas que habitan nuestros espacios de formación, hacen de la clase de educación física un espacio propicio para entroncar la formación inicial de los maestros a las nuevas tendencias de la educación y en especial a la de la educación física, puesto que es de imperiosa necesidad adaptarse a un futuro cambiante y altamente incierto.
El significado: es de suma importancia que los estudiantes le den el valor necesario y suficiente a este tipo de desarrollo académicos; por ello, la clase de educación física se debe desenvolver entre lo teórico y lo práctico, puesto que, una evanesciente metodología, daría paso a una decadente clase de educación física y surgiría del propósito de la clase meramente mecanicista, autoritaria y tradicional.

\section{Dice una estudiante de I semestre:}

"Me llamó la atención el trabajo práctico, ya que para realizar esta clase es muy necesario el tener disposición, estado físico y el enfoque que se le da a trabajar desde edades tempranas a jóvenes; me llamó la atención también es que se divide la clase en teórica y luego práctica y la integración del grupo"

Desde luego, diversos métodos han sido aplicados para la clase de educación física, pero no existe un único ni eficaz método, unos presentan más ventajas frente a otros, y en este sentido, lo mejor que se puede hacer es utilizarlos de forma combinada o conjuntamente.

El desempeño de la clase también depende de los espacios físicos donde se desarrolle. Los ambientes cambiantes son ideales, pues en ellos encontramos la posibilidad de enriquecer el trabajo pedagógico y de aprendizaje de los estudiantes y por su puesto, marcan la motivación tanto individual como colectiva.

Relación sistémica ${ }^{5}$ lo sistémico, permanece en el colectivo como lo armónico, lo integral, holístico, en este sentido, los estudiantes del programa, como Shyrley López estudiante de II semestre cuando enuncia que: "le encontré mucha relación con historia de la pedagogía pero se diferencia por la forma de darla de cada profesor era mas agradable la de historia de la pedagogía", o cuando expresa Jaime Andrés Ocampo, estudiante de III semestre,
"si, hay mucha relación. Ya que lo primero que vemos son la capacidades físicas, ya que en todo deporte, actividad física que vayamos hacer, estas capacidades determinan al deportista dependiendo de los factores energéticos y sensorimotrices de este. Estas capacidades están desarrolladas y determinadas, tanto por sucesos morfológicos, como psicológicos".

\footnotetext{
${ }^{4}$ La educación física es una disciplina pedagógica que contribuye significativamente a la estructuración de la personalidad del alumno, su creatividad, su socialización y el fortalecimiento de los aprendizajes escolares. Bonilla. V. Carlos. En didáctica de la educación física de base. Ed. Kinesis 1995.

${ }^{5}$ Desde este punto de vista, el sistema educativo no es un sistema independiente sino una institución que sirve para perpetuar las relaciones sociales de la vida económica y cultural de los sujetos. Díaz. V. Mario. Del discurso pedagógico: problemas críticos. Ed. Magisterio. 2001.
} 
En virtud de lo anterior, no basta con pretender formar un profesional eficiente y racional, si no que, también a de ser crítico en el sentido de fundamentar lo que postula, a la vez a de ser creativo al proporcionar alternativas de solución a los problemas planteados; debe ser reflexivo, en su capacidad de auto crítica y ético en su profesión cuando perfile y proteja un conjunto de valores y actitudes puestas por la cultura y nuestra idiosincrasia.

Por lo tanto, el futuro docente debe enriquecer los valores, las actitudes, los conocimientos, las habilidades intelectuales y las destrezas motoras que se reflejen el ser, en el hacer, en el saber y en el convivir.
En el segundo momento, se desarrollan entrevistas propias a la pregunta por la clase de educación física y el tercer momento, se desarrolla la estructura definitiva, se definen las categorías y se elabora la teoría, encontrando lo siguiente:

Se parte de los relatos del diario de campo y las entrevistas, los cuales se triangulan con la teoría formal encontrada, se exponen un número suficiente de categorías axiales, las cuales darán soporte a las categorías selectivas como son: Metodología, Innovación, La clase, la Actitud y El ambiente, entorno a las cuales girará la construcción del sentido del tercer momento o sea la reconfiguración.

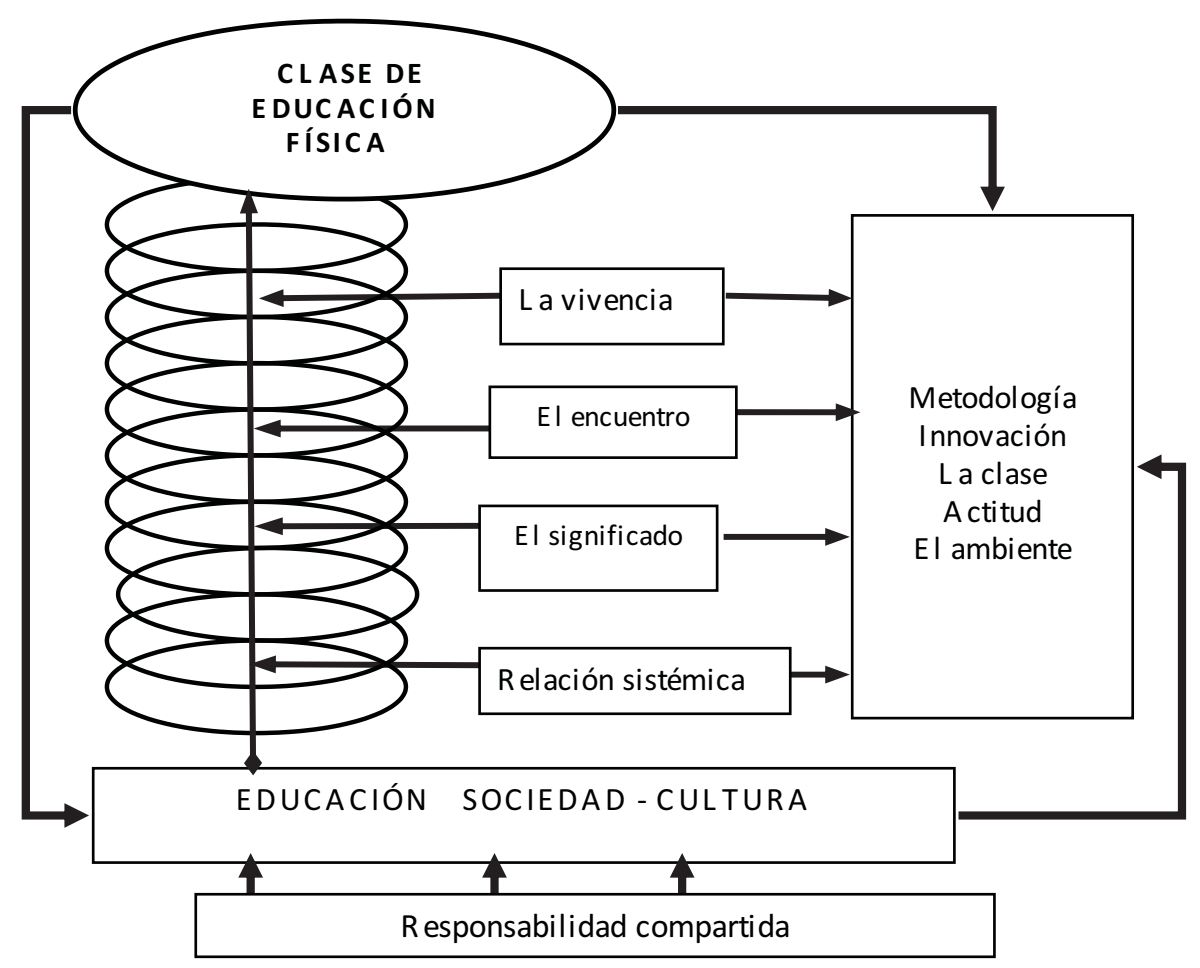

Del la estructura encontrada y puesta en evidencia en el cuadro anterior, podemos interpretar lo siguiente:

1. La metodología propuesta por el docente en la clase de educación física, es variada y rica en su hacer, como por ejemplo, un docente encuentra que para los estudiantes es bueno que "el estudiante puede y debe construir su propio esquema mental y a través de juegos predeportivos la misma metodología cuando lo desarrolle como licenciado", lo que nos lleva a pensar que el educar jugando, es una estrategia para mejorar la capacidad creadora del estudiante, es una de las concepciones teóricas del juego, de acción recreativa infundida en el hacer humano permitiendo el goce y el disfrute así como el placer.

De otro lado, "una metodología que atiende como proceso a un enfoque constructivista y que a su vez, orienta lo significativo del aprendizaje, puesto que garantiza que el deporte sea un medio para desarrollar habilidades, destrezas, mejores hábitos físicos y motores", nos enuncia un docente del programa en el campo profesional, siendo este aporte una de las características propias de la educación física emergente.

En tal sentido, el proceso enseñanza aprendizaje de los estudiantes basado en el eje deportivista, orienta la 
formación en dos vías: una en dirección al desarrollo de una condición física y la plena conciencia de la funcionalidad en pro del mejoramiento de la salud y elevando la calidad de vida las personas, lo que conlleva a desempeños eficientes en las actividades propias de la vida cotidiana, y la segunda vía, está dirigida hacia el rendimiento deportivo exclusivamente, lo que se ocupa de los métodos de entrenamiento, de la técnica y de la táctica en los deportes tanto individuales como colectivos.

Por otro lado, el desarrollo de la clase de educación física debe apalancarse en un buena planeación y organización de las actividades propias del quehacer educativo, por ello, es necesario sustentarse en el conocimiento de teorías vinculadas a los desarrollos del ámbito motor como forma de desarrollo personal.

Por lo tanto, la preparación de la clase pretende poder identificar y satisfacer las necesidades emergentes de la realidad vivida en clase y procurar abrir los espacios de interés en el ámbito pedagógico, didáctico e investigativo como proceso de mejoramiento de nuestra calidad educativa.

2. En cuanto a las innovaciones de la clase, estas permean el ámbito educativo en lo que concierne a la creatividad $^{6}$, es una de las características propias de la actividad socio cultural en el ámbito escolar. Esta categoría es inherente a cada persona, debe identificarlo por el dinamismo y ello depende del grado de motivación ${ }^{7}$ hacia el desarrollo de los procesos cognitivos desde el área.

En tal sentido, enuncia un docente del programa que "se les cultiva a los estudiantes la lectura en especial de artículos indexados o de portales reconocidos, dándoles las direcciones donde pueden leer $y$ consultar", donde la evolución metodológica se despliega de lo puramente funcional, dándole el carácter teórico al proceso metodológico, permitiendo que la creatividad ${ }^{8}$ y la innovación se constituyan en fuente de conocimiento para reconocer su valor en la compleja acción de investigación, organización, orientación y emancipación del hombre. Bien lo enuncia trigo en libro creatividad y motricidad, "es el valiosa -la creatividad-, no sólo como referencia al posible producto creado, sino a la acción misma de innovar, que supone un afán de superar, de mejorar, de optimizar cuanto nos rodea y a nosotros mismos" (1999:26)

De lo anterior, podemos decir que debemos hacer grandes esfuerzos para superar el estancamiento creativo ya que encontramos al profesor que "siempre se ha basado en lo mismo y no trae técnicas nuevas, es muy tradicionalista" afirma un estudiante de III semestre, ello nos muestra que todavía existen docentes poco innovadores y utilizan una metodología que nosotros denominamos educación física dominante, la cual consiste en que sus contenidos programáticos son extraídos de los manuales de técnicas deportivas o textos de publicación general, cayendo en una educación tradicionalista o tecnocrática.

El siempre lo mismo, es una categoría que surge del proceso del dejar hacer, de la negociación de los procesos escolares y que los estudiantes perciben cuando el docente no planea su actividad: los escenarios de clase son los mismos, el proceso metodológico es igual, etc, lo que da a entender que para algunos docentes, la creatividad como esa capacidad humana que poseemos, se diluye en la miscelánea de la facilidad y el conformismo para transmitir y profundizar en los conocimientos. Un estudiante nos comenta que "si nos ponemos a pensar, para nosotros los estudiantes relativamente sin experiencia en el campo, casi todo seria innovador, pero en sí, el profe no tienen ningún método innovador aparte de la forma que da la clase, que para mi puede no ser la correcta".

Pero encontramos aquel docente que "es recreativo y no tan metódico y cualquier tipo de innovación es agradable e indispensable para nuestra formación", dice un estudiante de II semestre. Asumir la creatividad como proceso de formación humana, es permitirle poner en juego todas las características de una persona creativa, que le sirva de soporte para fomentar los atributos que los seres humanos poseemos y que se desligan de la puesta en escena de nuestra creatividad.

3. En relación con la clase, encontramos que, para los estudiantes y tal como lo enuncian "Esta clase la vivo

\footnotetext{
${ }^{6}$ Siguiendo a Trigo, la creatividad es una capacidad humana que en mayor o menor medida todo el mundo posee; lo que para este estudio es de relevancia ya que en algunas reuniones y lecturas hemos estado en total concordancia con la autora en cuanto a que los docentes y ahora los futuros docentes deben tener muy clara esta concepción, ya que es de suma importancia mantener esa capacidad en potencia. Creatividad y motricidad. 1999.

${ }^{7}$ La motivación debe ser atribuida a factores internos como externos, aunque en muchas ocasiones ambos operan en variadas situaciones, lo más deseable e importante de estas fuentes es la motivación intrínseca. Berbesi. Hector. J. XI congreso nacional de educación física. Bogota. 1990.

${ }^{8}$ Se entiende la creatividad como la capacidad que todo sujeto tiene de aprovechar las experiencias vividas y con ellas formar patrones originales que anteriormente no habían sido planteados o logrados por él. Lora. R. Josefa. La educación corporal. Ed. Paidotribo. Barcelona 1991.
} 
al $100 \%$ y doy lo mejor puesto que me brinda alegría y entretenimiento", una concepción muy recreativa de la clase, para lo cual, la recreación también es un medio para el aprendizaje, o, "La vivo intensamente, juego y siempre trato de disfrutarla al máximo" este enfoque desde la perspectiva del juego, colabora en el proceso de enseñanza aprendizaje ya que tiene un alto grado de contenido motivacional, favoreciendo la participación del estudiante permaneciendo en el tiempo como proceso performance cultural y es de fácil aplicación por parte del maestro. En este sentido, la motivación determina en parte el éxito de la clase, permitiendo el desarrollo de los objetivos propuestos de manera positiva y a su vez fortalezca la acción colectiva.

Esta mirada llena de significado vivencial de la clase de educación física, se acerca a uno de los principios del aprendizaje que bien lo expresa Napoleón Murcia y otros en el texto de escuela de iniciación deportiva citando a pozo (1994) "un aprendizaje es significativo cuando puede incorporarse a las estructuras del conocimiento del sujeto, es decir, cuando el nuevo material adquiere significado para el mismo a partir de su relación con conocimientos anteriores", lo que le da sentido a nuestras prácticas curriculares en educación física, cuando una docente de nuestro programa afirma que "Soy conocedora que todo aprendizaje se hace significativo siempre que uno vea las cosas mas simples en un mundo de cosas grandes, amo lo que hago". En este sentido, la clase de educación física se proyecta como un espacio relacional en el cual los estudiantes y docentes no se conforman con lo desarrollado en la clase, sino que anhelan o buscan satisfacer sus deseos de aprendizaje y de proyección para su vida.

De otra parte, esta mirada de proyección y significado, permite aproximarnos a los procesos de identidad del sujeto, ya que, el proyectarse implica ir más allá, ser yecto (trascendencia) en todas aquellas relaciones establecidas por el entorno o mundo vital del sujeto (en Jaramillo: 2005; 131)

4. La actitud frente a la clase, se refiere al desempeño del docente y estudiante en relación con el nivel de compromiso e interés con el que asuma su rol. "Pienso que les falta mayor compromiso y responsabilidad para las actividades que se les asigna", dice un profesor del programa, para quien el compromiso y la responsabilidad es fuente de actuación consciente de los estudiantes en el proceso de enseñanza y de la forma como se desarrollan las actividades propias del acto educativo.

El acto educativo, encierra una gama de elementos que se pueden considerar como una estructura compleja que se va develando y complementando a medida que se avanza en el desarrollo del mismo acto, hecho que se determina por el grado de compromiso y de actitud de los actores del proceso puestos en el ejercicio de su oficio.

"En este momento estamos conociendo al profesor, pero me parece que tiene muy centrado a que vino, no es de los que dice hagan y yo los evalúo... también es muy correcto en sus cosas, nos da el cronograma de lo que hay que hacer y nosotros lo hacemos poro con su ayuda"

En este sentido, la clase de educación física encuentra eco en el proceso de evaluación y en la coresponsabilidad que tienen los actores de ella; la evaluación se presenta cuando el docente orienta y permite la ejecución de las tareas asignadas para la clase, por ello, la evaluación por actitud pone su atención en el modo de actuar, de desempeño y de ello dependerá su resultado; es decir, lo que el estudiante ha producido, más no se centra en lo aptitudinal, que hace referencia al resultado y la eficiencia en el logro de la clase.

La participación de todos los actores en el desarrollo de la clase de educación física es de vital importancia, ya que ésta debe ser efectiva, asumida como un compromiso permanente, como un asunto que refleja la fidelidad y la intencionalidad del progreso que ellos hacen desde cada sesión de trabajo.

5. En cuanto a los ambientes, podemos decir que, en general se presenta "con variedad, dinámica, organización y estructura puesto que se están formando futuros licenciados", dice un docente del programa, y a su vez, "es muy vacano porque es muy diferente a las demás clases, muy dinámica, el ambiente en la clase es muy bueno porque todos compartimos y nos comunicamos sin egoísmo.", enuncia un estudiante de III semestre del programa.

De manera que, los ambientes educativos hacen parte inherente del mismo desarrollo de aprendizaje, los cuales hacen posible la realización de los procesos y actividades propuestas, contribuyendo al posicionamiento y estructuración de la clase desde una perspectiva humanista.

No podemos olvidar que el docente es el responsable de la operacionalización de la clase; debe procurar organizar el desarrollo de los contenidos de acuerdo a los espacios que han de ser correspondientes con los intereses y necesidades de cada grupo.

\section{A manera de viaje y conclusión}


El concepto de clase de educación física que tienen los estudiantes del programa de Licenciatura en Educación Física de la Universidad del Quindío, gira en torno a lo siguiente:

1. Es importante rescatar que un primer hallazgo es que no existe un concepto único por el cual se denomine la clase de educación física, sin embargo, nos deja entre ver este estudio, que para los estudiantes del programa de Licenciatura en educación física de la Universidad del Quindío esta posible concepción está relacionada con su didáctica y práctica pedagógica docente para la cual se están formando.

2. En consecuencia la clase de educación física sería pues, un espacio de desarrollo pedagógico y didáctico donde intervienen las actitudes, deseos y sentimientos puestos en acciones individuales con fines colectivos.

3. De otra parte, la clase de educación física depende de la creatividad, el entusiasmo y el respeto por lo que se hace, que medie entre la formación y el aprendizaje como proceso de cualificación del hombre en su desarrollo motor; es acción en movimiento, lo que quiere decir es que existe un verdadero enriquecimiento del hombre por medio de la vivencia de su propio cuerpo.

Finalmente, queda abierto el tránsito para el nuevo viaje en el vasto mundo de la educación física, que posiblemente este en el orden del ser, del conocer, del saber, del hacer y de los nuevos horizontes que merecen el despliegue de todos.

\section{BIBLIOGRAFÍA}

Berbesi. H. J. (1990) XI congreso nacional de educación física. Bogotá.

Bonilla, C. Pedagogía y Cultura Física. Editorial Kinesis. Armenia. 1995

Bonilla, C (2000) La cultura corporal de los adolescentes escolares. Neiva, Trilse Editores.

Díaz. M. (2001) Del discurso pedagógico: problemas críticos. Editorial Magisterio. Bogotá. D.C.

Jaramillo. L. (Diciembre 2001) La Tensión entre lo Global y lo Local. Revista Digital. Cinta de Moebio No. 12.. F. Ciencias Sociales. U. Chile. http://rehue.csociales.uchile.cl/publicaciones/moebio/12/frames08.htm

Jaramillo. L. Hurtado. D. (Mayo 2005) Jóvenes e imaginarios de la Educación Física. Un estudio Comprensivo en la Ciudad de Popayán. Diseño grafico impresiones. Universidad del Cauca. Popayán. C.

Le Boulch, J. (1993). Releyendo a Jean Le Boulch. En: Revista Kinesis, Armenia. Edición Nº 10.

Ley General de Educación (115). (1994). Editorial Magisterio. Bogotá. D.C

Legislación deportiva. Ley 181 (2001). Coldeportes Nacional, Editorial Kinesis, Armenia. Q.

Lineamientos Curriculares. Educación Física, Recreación y Deportes. (Julio de 2000) Ministerio de Educación Nacional, Editorial Magisterio. Bogotá.

López. J. Zuluaga. L. (2005) Imaginarios del joven frente a la clase de Educación Física. Informe de Investigación; p: 243. Colciencias. Editorial Kinesis. Armenia, Q.

López. J. Zuluaga. L. (2007) Funcionalismo y Banalidad, Evanescencia y Alteridad. Centro de Publicaciones Universidad del Quindío. Armenia. Q.

Lora. R. Josefa. (1991)La educación corporal. Editorial Paidotribo. Barcelona.

Murcia, N y Jaramillo, L. (1998) La danza, factor de promoción ético-moral en jóvenes marginados de la Ciudad de Manizales. En: Revista Apunts $N^{\circ} 54$. Barcelona.

Trigo, E. (1999). Creatividad y Motricidad. Editorial Inde. España. 